\title{
El régimen diario del viento en la estación meteorológica de Santa Elena de Uairén (Venezuela)
}

The daily wind pattern at the Santa Elena de Uairén meteorological station (Venezuela)

\section{Sergio Foghin-Pillin}

sfoghin@hotmail.com

Código ORCID: 0000-0003-3690-8195
< Articulo aceptado en septiembre 2020

< Arbitrado en octubre 2020

< Publicado en enero 2021

\section{Universidad Pedagógica Experimental Libertador \\ Caracas - Venezuela}

Resumen

En este trabajo se rescatan los registros anemométricos horarios de la estación meteorológica de Santa Elena de Uairén, correspondientes a la serie temporal 1982-1991, los cuales no se encuentran digitalizados. Con base en dichos datos se describen las variaciones horarias de la velocidad y dirección del viento a lo largo del año. Se comparan las condiciones de la serie 1982-1991 con algunas características de la circulación local del viento en el antiguo emplazamiento de la estación, basadas en la serie temporal 1971-1980. Se evidencian diferencias significativas entre ambos emplazamientos. Se interpretan las variaciones diarias del viento en el marco de las condiciones topográficas locales. Se resalta la urgencia de la recuperación de los registros climatológicos no digitalizados y la importancia de los estudios de las variaciones horarias de la dirección y velocidad del viento, para diferentes aplicaciones en ingeniería y ciencias ambientales.

Palabras clave: Meteorología, registros anemométricos, vientos locales, velocidad del viento, dirección del viento

Abstract

In this work, the hourly anemometric records of the Santa Elena de Uairén weather station, corresponding to the 1982-1991 time series, which are not digitized, are rescued. Based on these data, the hourly variations in wind speed and direction throughout the year are described. The conditions of the 1982-1991 series are compared with some characteristics of the local wind circulation at the former site of the station, based on the 1971-1980 time series. Significant differences are evident between both sites. The daily variations of the wind are interpreted within the framework of local topographic conditions. The urgency of the recovery of the nondigitized climatological records and the importance of studies of the hourly variations of wind direction and speed for different applications in engineering and environmental sciences are highlighted.

Keywords: Meteorology, anemometric records, local winds, wind speed, wind direction 


\section{INTRODUCCIÓN}

El análisis climatológico de los regímenes anemométricos diarios, presenta interés por cuanto permite conocer la evolución horaria de las curvas de velocidad y las variaciones que pueden ocurrir en la dirección del viento a lo largo del día y durante el año, incluyendo las condiciones de calma. Los resultados de tales análisis pueden presentarse por medio de polígonos, rosas de viento o simples curvas y tablas de datos. Estos registros permiten identificar y caracterizar sistemas de vientos locales, de ciclo diario, como las brisas de mar-tierra (Miller, Keim, Talbot y Mao, 2003; Simpson, 1994), en regiones litorales, y las brisas de valle-montaña, en áreas con relieve montañoso (Barry, 1992; Serafin y Zardi, 2010).

Estas circulaciones locales han sido poco investigadas en el territorio venezolano, aunque revisten notable importancia en los estudios de impacto ambiental requeridos en diferentes áreas de la ingeniería, para la estimación de tasas de erosión y de evapotranspiración, así como para el desarrollo de complejos industriales que generen contaminantes, tanto gaseosos como particulados (petroquímicas, cementeras), especialmente para el cálculo de la altura de chimeneas conjuntamente con la estratificación térmica de la capa límite-, horarios de descargas a la atmosfera (inmisiones), a la vez que para la localización de rellenos sanitarios $u$ otras actividades que puedan generar olores desagradables, tales como granjas avícolas y porcinas, entre otras (Henry y Heinke, 1999).

A escala local, los patrones diarios de circulación atmosférica también son importantes en el diseño de sistemas de ventilación, aprovechamiento de fuentes de energía renovables, diseño y orientación de pistas de aterrizaje, así como otras aplicaciones ingenieriles y arquitectónicas (Jiménez, GonzálezRouco, Montávez, García-Bustamante y Navarro, 2009).

Los vientos locales de ciclo diario influyen así mismo en la dispersión de semillas de especies anemocóricas (Escobar, Silveira y Morellato, 2018), microrganismos potencialmente patógenos, como bacterias y virus (Burnet y White, 1982; Hospodowsky et. al., 2012), así como esporas y polen, los cuales pueden constituir factores alergénicos (Benaim-Pinto, 1972; Ezike, Nnamani, Ogundipe y Adekanmbi, 2016), además de otros aerosoles contaminantes (Hafner y Hites, 2006; Simpson, 1994).

Por otra parte, la recuperación de series de datos de décadas pasadas, frecuentemente no digitalizadas, permitirá futuras comparaciones en investigaciones sobre las posibles alteraciones derivadas del cambio climático global (Pino y Espinosa, 2001), las cuales podrían convertirse en factores de interés ecológico (Kaczmarek et al, 2016).

Específicamente en el caso de la ciudad de Santa Elena de Uairén, la 
expansión que actualmente experimenta el centro poblado hacia el sur de su emplazamiento original, a lo largo del curso del río Uairén y hacia el área del aeropuerto, donde se localiza la estación meteorológica, incrementa el interés de este tipo de estudios ambientales por las posibles alteraciones que podría generar sobre la circulación local, efectos que han sido evidenciados en investigaciones efectuadas en otros países (Kitada, Okamura y Tanaka, 1998).

La mencionada población, capital del municipio Gran Sabana del estado Bolívar, se localiza al sureste de Venezuela, en la provincia fisiográfica de la Guayana venezolana, ramal del Usari-Uará de la región de la Sierra de Pacaraima (Freile, 1962). La localidad dista unos 20 kilómetros de la línea limítrofe con Brasil (Figura 1).



Figura 1. Fragmento del mapa topográfico a escala 1:100.000, de Santa Elena de Uairén, estado Bolívar, Venezuela (Dirección de Cartografía Nacional, 1977). El aeropuerto se observa al SSW de la población de S. Elena, próximo a la confluencia de la quebrada Aserradero y el río Uairén. 
La geología regional está conformada por las secuencias de rocas sedimentarias precámbricas, pertenecientes a la Formación Roraima, las cuales afloran en la orografía local, mientras que en las márgenes de los cursos de agua aparecen los depósitos de aluviones cuaternarios (Schubert y Huber, 1989). El área pertenece a la cuenca alta del río Caroní, del cual es afluente el río Cuquenán, que a su vez recibe las aguas del río Uairén, en cuyo curso medio se encuentra ubicada la estación meteorológica sinóptica de Santa Elena.

Según las normales climatológicas del lapso 1961-1990, de la misma estación (SMFAV, 1993), la temperatura media anual es de $21,4{ }^{\circ} \mathrm{C}$, mientras que las medias térmicas anuales máxima y mínima son de $28,1{ }^{\circ} \mathrm{C}$ y $16,6{ }^{\circ} \mathrm{C}$, respectivamente. $\mathrm{El}$ monto pluviométrico anual promedia 1.694 milímetros y la evaporación media anual alcanza los 862 milímetros, presentándose déficit de humedad solamente durante los meses de enero a marzo.

La estación meteorológica de Santa Elena de Uairén está identificada con el serial internacional 80462. Fue fundada como estación climatológica en 1940 y posteriormente reclasificada como estación sinóptica a partir de agosto de 1949, en las coordenadas: latitud 4'36'09" $\mathrm{N}$; longitud 6106'57"W; altitud 907 m/nm, aproximadamente. En 1981 fue reubicada en predios del aeropuerto, en las coordenadas: latitud $4^{\circ} 33^{\prime} 05^{\prime \prime} \mathrm{N}$; longitud $61^{\circ} 08^{\prime} 21^{\prime \prime} \mathrm{W}$; altitud
$896 \mathrm{~m} / \mathrm{nm}$, adyacente al extremo sureste de la pista de aterrizaje, donde se encuentra hasta el presente, distante unos 6,5 kilómetros al SSW de su antigua localización, en el centro del poblado.

La estación meteorológica actual se encuentra en una posición de valle amplio, emplazada sobre la margen derecha del río Uairén, rodeada de montañas distantes unos 6-7 kilómetros hacia el norte, este y sur (Sierra de Pacaraima). Las mayores elevaciones se encuentran al oriente de la estación, con altitudes que superan los 1.300 metros en los relieves tabulares del cerro Maracmotá. Hacia el oeste se extiende el valle alto del río Uairén, con altitudes entre 900 y 1.000 metros, aproximadamente (Figura 1).

En su anterior ubicación, la estación se encontraba a unos 1.400 metros al sur del cerro Akurimá, de poco más de mil metros de altitud, a un kilómetro aproximadamente de las estribaciones orientales del cerro Pacaiguariyén, que rodean el centro de la población por el noroeste, oeste y suroeste. Hacia el sureste del antiguo emplazamiento se levanta el cerro Maracmotá, ya mencionado, mientras que hacia el noreste se abre el curso bajo del río Uairén (Figura 1).

Con base en las características expuestas, el objetivo de la presente investigación se centró en calcular y describir las variaciones diarias de la dirección y velocidad del viento, así como los porcentajes de calmas mensuales (enero, abril, julio y septiembre), a partir de la serie de 
registros del período 1982-1991, para comparar, en líneas generales, dichas condiciones con los resultados obtenidos en una anterior investigación (Foghin-Pillin y Reyes, 1997), en la cual se analizaron los registros de la serie 19711980, datos procedentes de la estación de Santa Elena de Uairén en su antigua localización, antes descrita, en pleno casco urbano del centro poblado.

En la investigación se planteó así mismo el propósito de rescatar los registros anemométricos de la serie 19821991, datos que no se encuentran digitalizados, motivo por el cual su preservación podría verse comprometida.

\section{MATERIALES Y MÉTODOS}

Los datos utilizados en esta investigación se obtuvieron de las formas 0027 de registros mensuales del Servicio de Meteorología de la Fuerza Aérea Venezolana (SMFAV), en las que aparecen asentadas manualmente, por días y por horas, las observaciones de dirección (grados acimutales) y velocidad del viento (metros por segundo) de la estación de Santa Elena de Uairén, ambas variables obtenidas de la evaluación de las bandas registradoras diarias del anemocinemógrafo $\bigcirc$ anemógrafo universal (Figura 2). Dicha información, como se ha apuntado, no se encuentra respaldada en soportes electrónicos y reposa en los archivos de la Sección de Climatología del SMFAV (Base Sucre, Maracay, estado Aragua).
Se computaron los datos de 120 formas SMFAV-0027, correspondientes a los doce meses de los años desde 1982 a 1991. El criterio aplicado para seleccionar dicho período se basó en la disponibilidad y la calidad relativa de los registros, ya que en esta serie temporal sólo aparecen como faltantes los registros de velocidad del viento del mes de noviembre de 1986 y los registros de dirección de febrero de 1982 y enero de 1986. Por otra parte, la década en cuestión es la primera obtenida tras el cambio de ubicación de la estación.

Se consideraron las faltas señaladas para los cálculos de las velocidades medias respectivas y no se aplicó ninguna técnica de recuperación de datos faltantes, por considerarse que no afectan significativamente los resultados (Barros y Schmidt, 1988; Guevara, 1987). Se calcularon las velocidades medias horarias (media aritmética de las 00 a las 24) para cada mes e igualmente se computaron las direcciones prevalecientes para cada hora, seleccionándose la dirección o direcciones que presentan la mayor frecuencia. Se calcularon también los porcentajes medios de calma para los meses de enero, abril, julio y septiembre.

$\mathrm{Se}$ describen los resultados de las variaciones mensuales de velocidades medias y direcciones por horas, y se presentan las curvas horarias de velocidad para cada mes y para los promedios anuales 


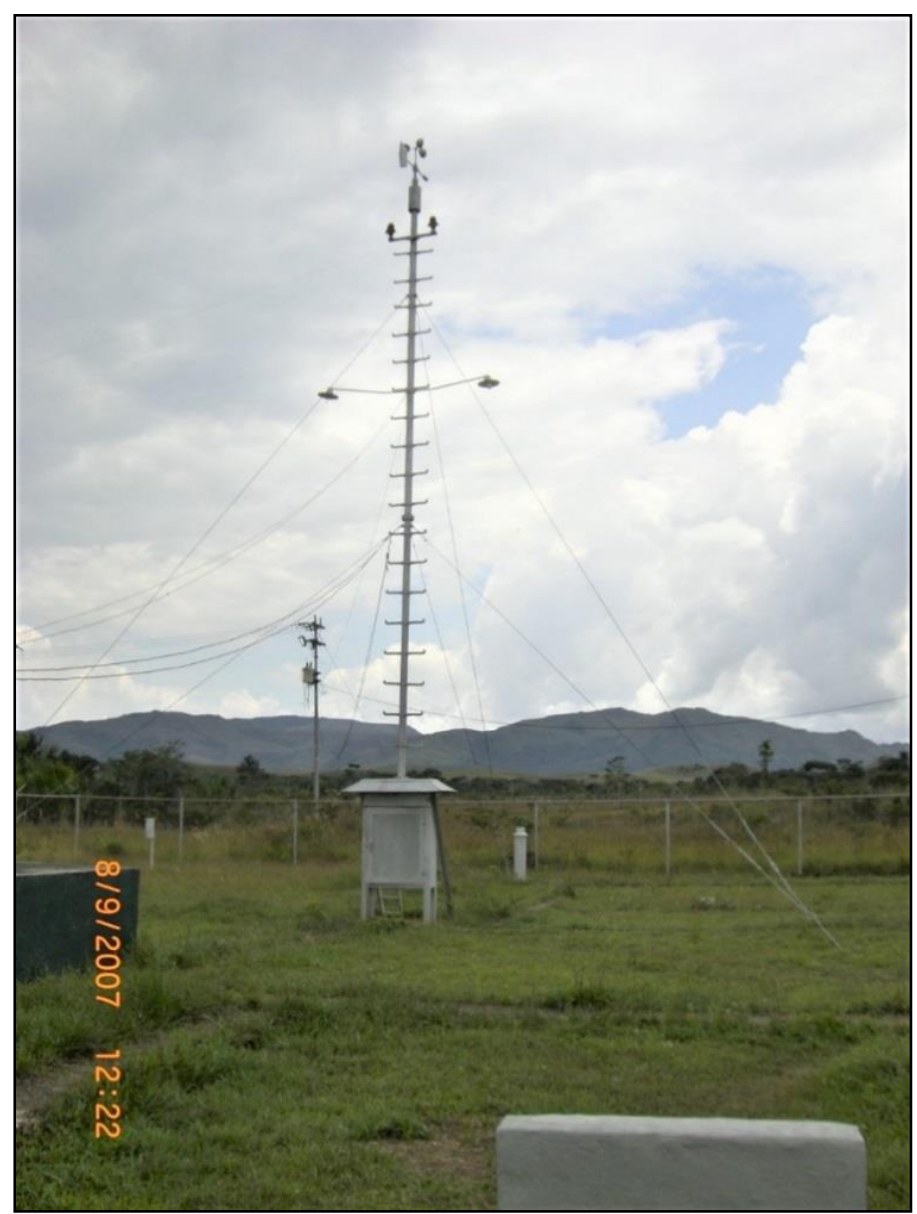

Figura 2. Vista del anemógrafo universal de la estación meteorológica sinóptica de Santa Elena de Uairén. Al fondo, en dirección este, se levantan las estribaciones occidentales del cerro Maracmotá, distantes unos cinco kilómetros. (Fotografía: V. M. Reyes, 2007).

\section{RESULTADOS}

Seguidamente, se describen la curva anual y las curvas mensuales de variación de la velocidad media horaria del viento en la estación de Santa Elena de Uairén, para el período 1982-1990. Del mismo modo, se describen las variaciones horarias de la dirección prevaleciente del viento.
Variaciones de la velocidad media horaria del viento

Variación de la velocidad media horaria de la década (1982-1991)

Para la variación media horaria de la velocidad del viento durante el lapso 1982-1991 (ver Figura 3) el análisis de los datos revela una curva en forma de campana simétrica, la cual alcanza su culminación a las 14 horas (Hora Legal Venezolana $=\mathrm{HLV}$ ), con una velocidad media de $3,5 \mathrm{~m} / \mathrm{s}$. La 
variación de la velocidad media es casi nula entre las $20 \mathrm{HLV}$ y las $08 \mathrm{HLV}$, con valores cercanos a $1,0 \mathrm{~m} / \mathrm{s}$, presentando luego un gradiente fuerte hasta la hora de culminación y luego descender con pendiente igualmente acusada hasta las $19 \mathrm{HLV}$. La amplitud de la curva es de $2,7 \mathrm{~m} / \mathrm{s}$.

Cabe destacar que esta onda diaria media es bastante similar, en su forma, a las de otras estaciones localizadas en diferentes regiones del territorio venezolano (Mene Grande, Maiquetía, Barcelona), aunque los valores medios y las amplitudes varían notablemente de región a región, como resulta esperable (Foghin-Pillin, 2002).

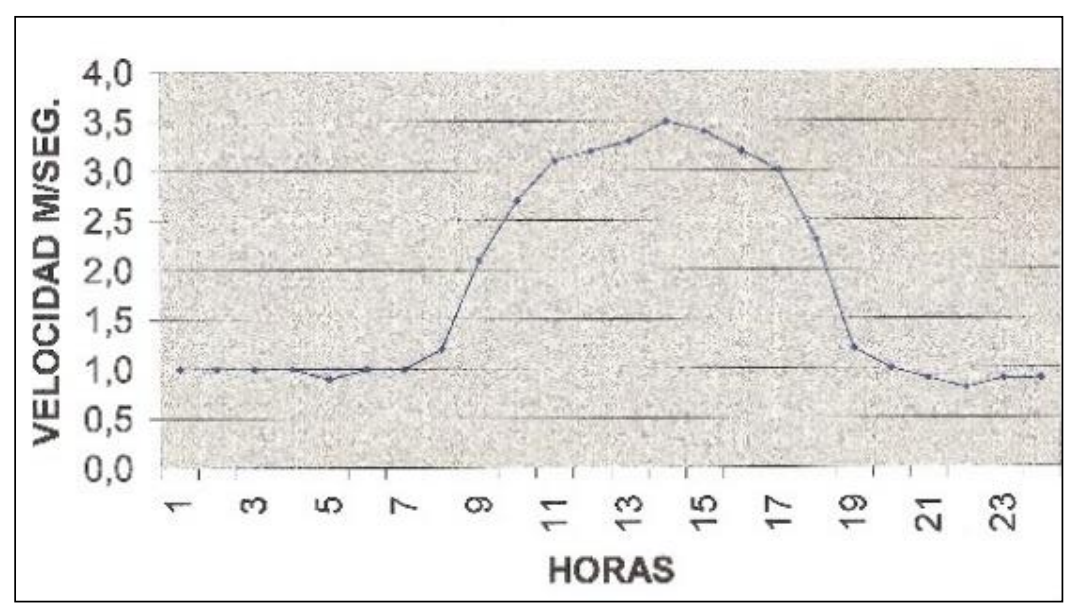

Figura 3. Variación media horaria-anual de la velocidad del viento 1982-1991. Fuente: elaboración propia.

Variación de la velocidad media una segunda, con los mismos valores, a horaria del mes de enero (1982- las 14-15 HLV, separadas por leve 1991) flexión a las 12-13. El rango medio de la

Esta onda alcanza una primera culminación a las $11 \mathrm{HLV}$, con $3,2 \mathrm{~m} / \mathrm{s}$ y curva para el mes de enero es de 2,1 $\mathrm{m} / \mathrm{s}$. (Figura 4). 


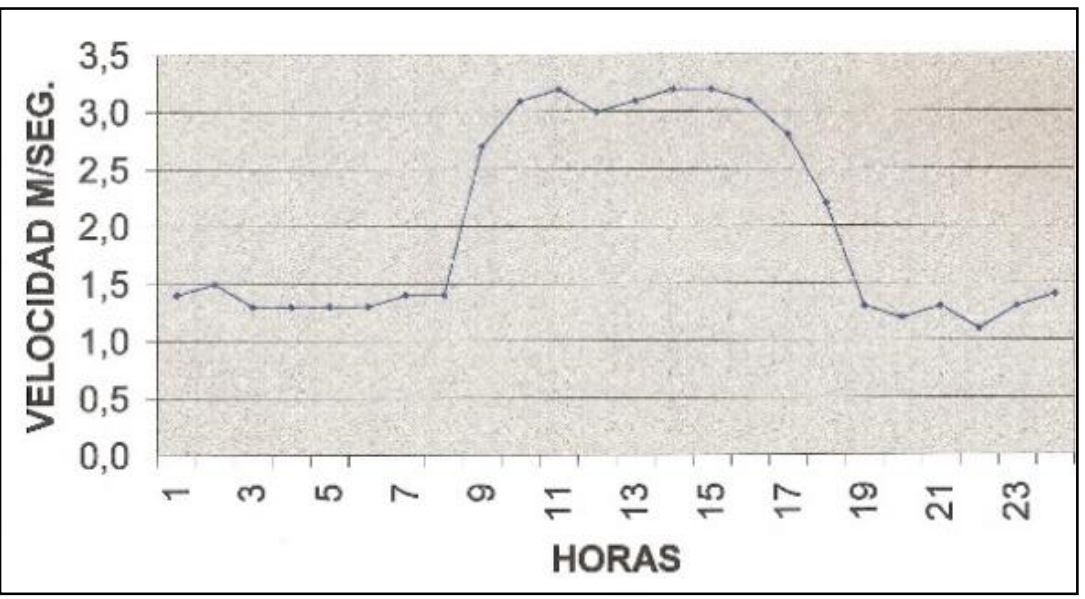

Figura 4. Variación horaria de la velocidad del viento en el mes de enero.. Fuente: elaboración propia.

Variación de la velocidad media horaria del mes de febrero (19821991)

La curva de velocidad media horaria de febrero presenta una primera cúspide a la 10-11 HLV, con medias de $3,2 \mathrm{~m} / \mathrm{s}$, seguida de una flexión que alcanza los $2,9 \mathrm{~m} / \mathrm{s}$, para alcanzar la máxima principal a las 14 $\mathrm{HLV}$ con $3,3 \mathrm{~m} / \mathrm{s}$ y última cúspide a las $17 \mathrm{HLV}$, tras la cual la curva alcanza abruptamente $1,5 \mathrm{~m} / \mathrm{s}$ en sólo dos horas. El ascenso matutino ocurre igualmente con una fuerte pendiente que pasa de 1,6 a $3,0 \mathrm{~m} / \mathrm{s}$ de las 8 a las 9 HLV. (Figura 5).

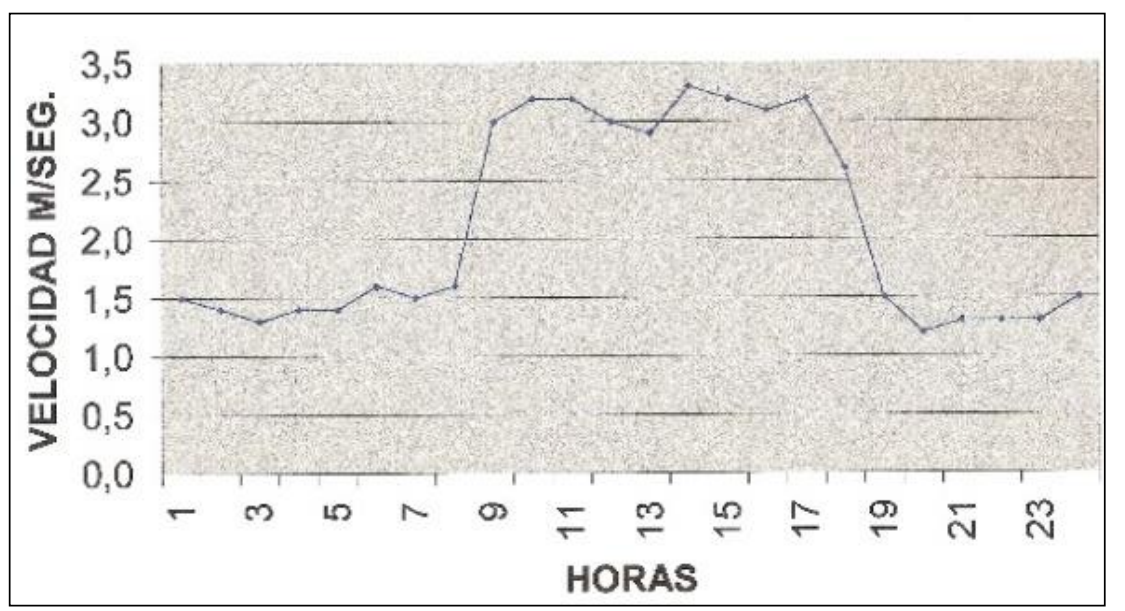

Figura 5. Variación horaria de la velocidad media del viento en el mes de febrero. Fuente: elaboración propia.

Variación de la velocidad media horaria del mes de marzo (19821991)
La onda de velocidad media horaria del mes de marzo presenta una amplitud de $1,8 \mathrm{~m} / \mathrm{s}$, con mínimas a las 06 y a las $20 \mathrm{HLV}$ y una cúspide que 
alcanza los $3,3 \mathrm{~m} / \mathrm{s}$ a las $11 \mathrm{HLV}$. gradientes fuertes en el ascenso entre Destaca la meseta de las 14 a las 17, las 08 y las $09 \mathrm{HLV}$, igual que en el con $3.1 \mathrm{~m} / \mathrm{s}$ tras una ligera flexión a las descenso entre las 18 y las $20 \mathrm{HLV}$. $13 \mathrm{HLV}$ (Figura 6). La curva presenta

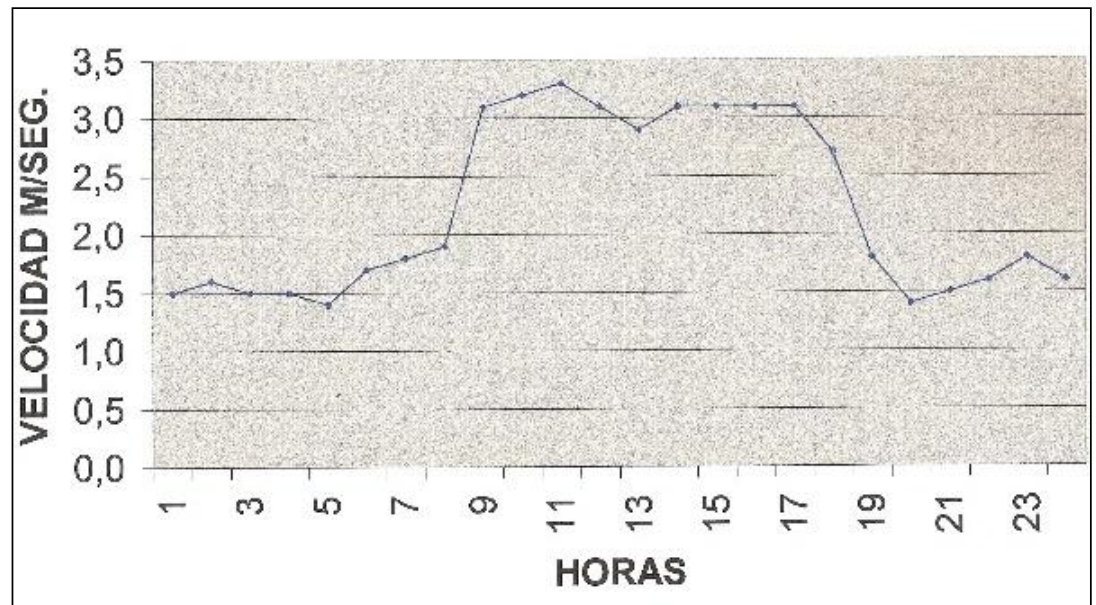

Figura 6. Variación horaria de la velocidad media del viento en el mes de marzo. Fuente: elaboración propia.

Variación de la velocidad media luego iniciar el descenso con horaria del mes de abril (1982-1991) pendiente fuerte sobre todo desde las

La curva del mes de abril muestra una primera cúspide a las $12 \mathrm{HLV}$, con un valor medio de $3,1 \mathrm{~m} / \mathrm{s}$, seguida de una leve flexión y luego la máxima del día, con $3,4 \mathrm{~m} / \mathrm{s}$ a las $14-15 \mathrm{HLV}$, para 18 hasta concluir el ciclo, con valores que se mantienen siempre por encima de $1,0 \mathrm{~m} / \mathrm{s}$. La pendiente de la curva en su segmento matutino es máxima entre las 8 y las 09 HLV. (Figura 7).

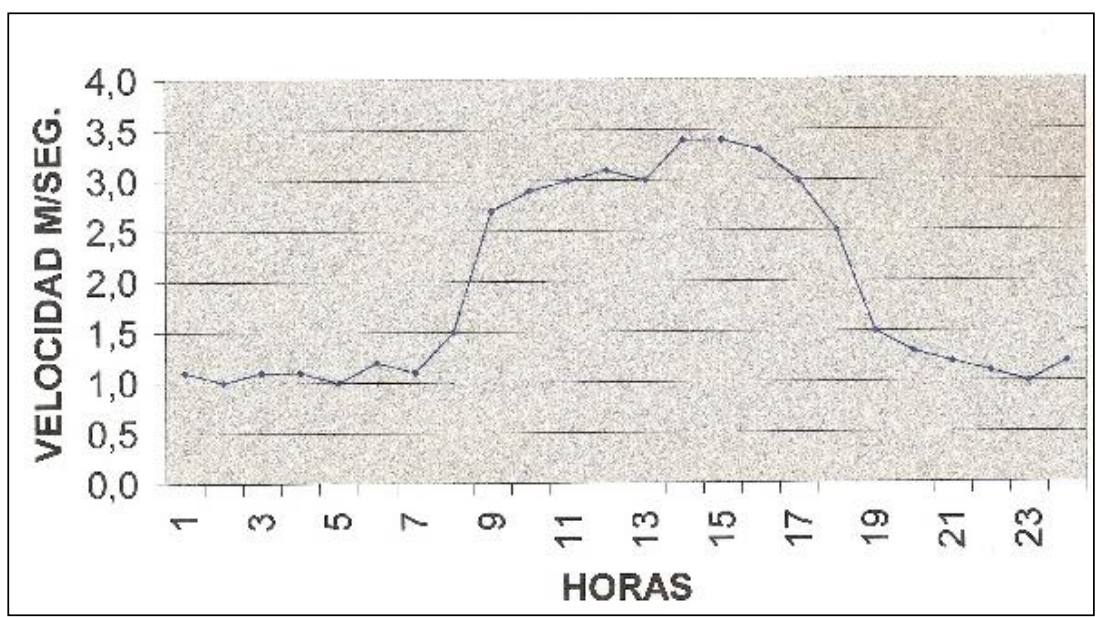

Figura 7. Variación horaria de la velocidad media del viento en el mes de abril. Fuente: elaboración propia. 
Variación de la velocidad media horaria del mes de mayo (1982-1991)

La curva horaria media del mes de mayo asume una forma más regular que las anteriormente descritas, por cuanto no presenta la flexión de las horas centrales, lo cual constituye una diferencia significativa sumada al pico de mayor velocidad que en este mes ocurre a las $14 \mathrm{HLV}$ con un valor medio de $3,7 \mathrm{~m} / \mathrm{s}$. Los menores valores, de 0,7$09 \mathrm{~m} / \mathrm{s}$, entre las 23 y las $07 \mathrm{HLV}$, determinan también que esta curva presente mayor amplitud que las anteriores, con un rango diario medio de $3,0 \mathrm{~m} / \mathrm{s}$. (Figura 8 ).

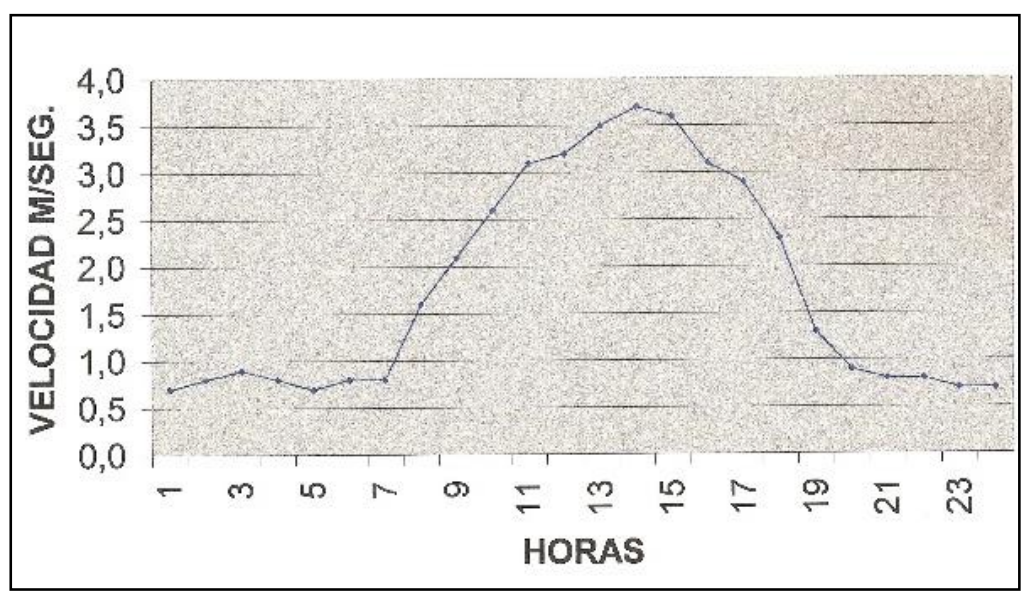

Figura 8. Variación horaria de la velocidad media del viento en el mes de mayo. Fuente: elaboración propia.

Variación de la velocidad media horaria del mes de junio (1982-1991)

Tras un rápido ascenso de la velocidad media entre las 08 y las 11 $\mathrm{HLV}$, con valores que pasan de 0,8 a $3,2 \mathrm{~m} / \mathrm{s}$, la curva correspondiente al mes de julio vuelve a mostrar una inflexión, aunque de sólo $1,0 \mathrm{~m} / \mathrm{s}$ a las
$12 \mathrm{HLV}$, para culminar a las $14 \mathrm{HLV}$ con un valor medio de $3,8 \mathrm{~m} / \mathrm{s}$, para luego alcanzar los mínimos valores medios desde las 23 hasta las 01 HLV, determinando una amplitud diaria media de $3,2 \mathrm{~m} / \mathrm{s}$, que representa el mayor rango desde enero. (Figura 9). 


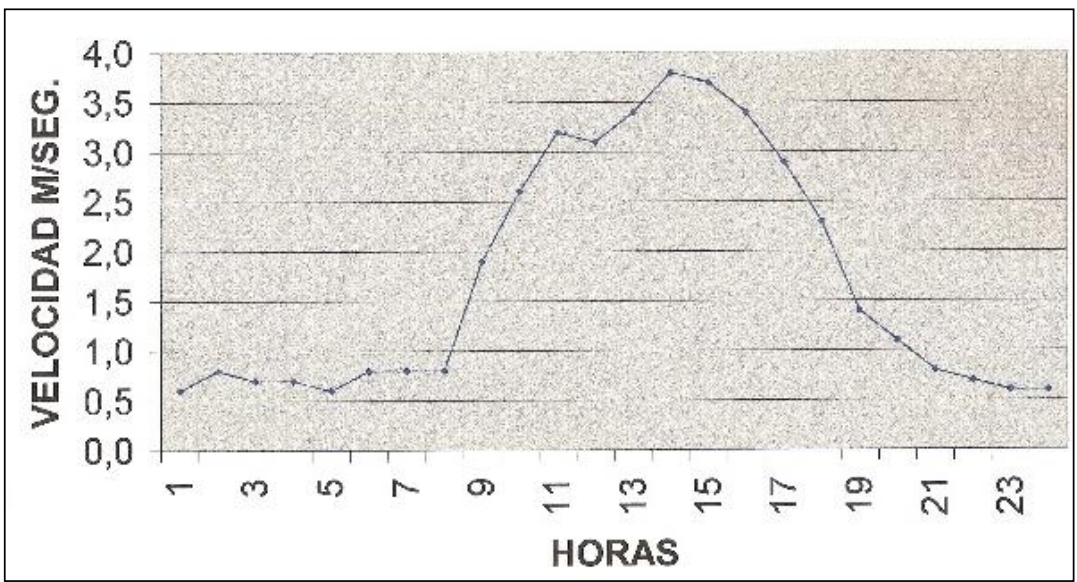

Figura 9. Variación horaria de la velocidad media del viento en el mes de junio. Fuente: elaboración propia.

Variación de la velocidad media horaria del mes de julio (1982-1991)

La curva del mes de julio describe una campana notablemente simétrica en las horas centrales del día, entre las
08 y las $21 \mathrm{HLV}$. El rango diario medio es de $3,1 \mathrm{~m} / \mathrm{s}$. Destaca la variación gradual entre las 11 y las $16 \mathrm{HLV}$. (Figura 10).

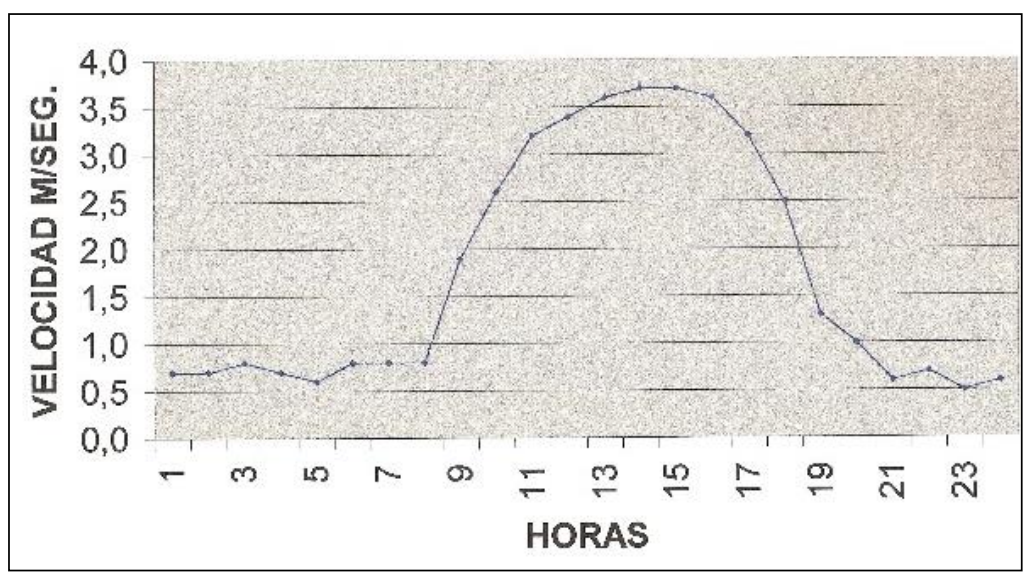

Figura 10. Variación horaria de la velocidad media del viento en el mes de julio. Fuente: elaboración propia.

Variación de la velocidad media horaria del mes de agosto (19821991)

Similarmente al caso del mes anterior, agosto muestra una curva de velocidad media notablemente regular, aunque con un rango diario poco mayor, que promedia $3,2 \mathrm{~m} / \mathrm{s}$, entre la máxima velocidad media, de $3,7 \mathrm{~m} / \mathrm{s}$, a las 14 y $15 \mathrm{HLV}$, y la mínima velocidad media a las $24 \mathrm{HLV}$, con 0,5 $\mathrm{m} / \mathrm{s}$. (Figura 11). 


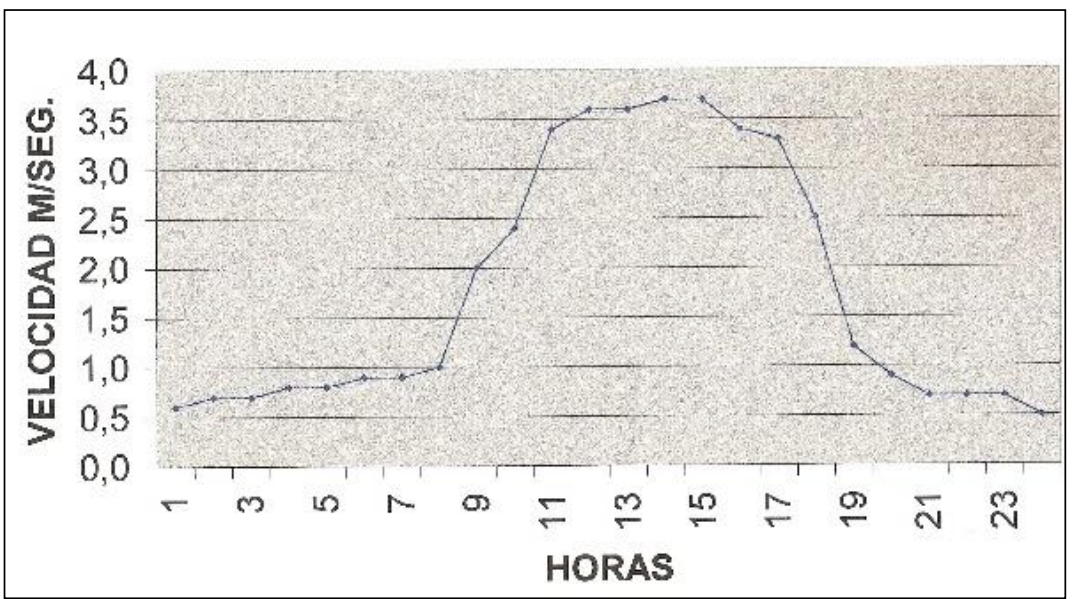

Figura 11. Variación horaria de la velocidad media del viento en el mes de agosto. Fuente: elaboración propia.

Variación de la velocidad media horaria del mes de septiembre (1982-1991)

Los valores medios de la curva del mes de septiembre parten de una mínima de $0,7 \mathrm{~m} / \mathrm{s}$ hasta la culminación de las 13 a las $15 \mathrm{HLV}$, con
$3,7 \mathrm{~m} / \mathrm{s}$. También en este caso la forma de la campana es notablemente regular. Similarmente a la mayor parte de los meses anteriores, se observa un leve aumento aislado hacia el final del ciclo diario (Figura 12).

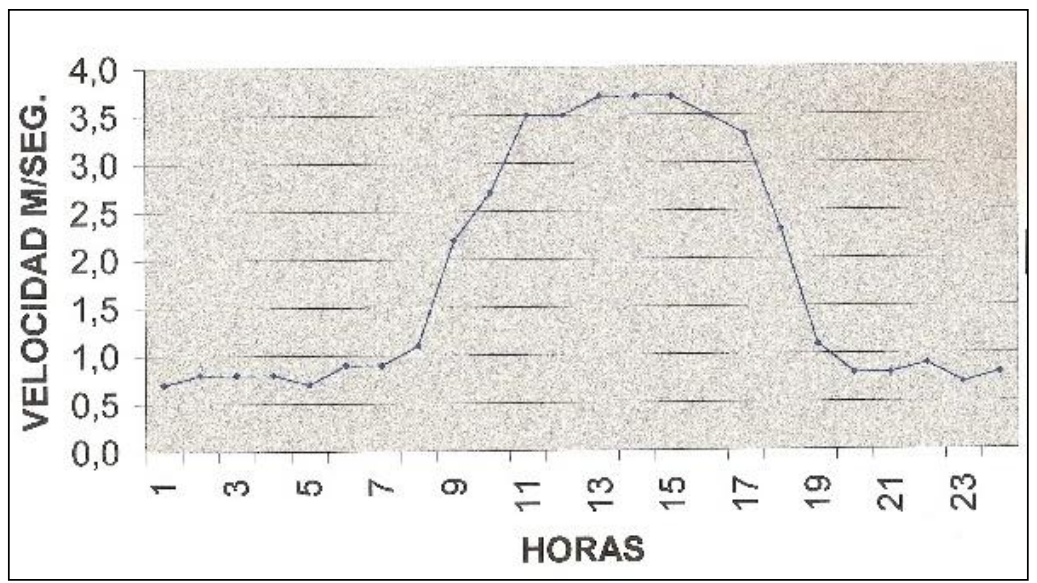

Figura 12. Variación horaria de la velocidad media del viento en el mes de septiembre. Fuente: elaboración propia.

Variación de la velocidad media horaria del mes de octubre (19821991)
La curva del mes de octubre muestra una forma de campana regular, interrumpida por un descenso de $0,1 \mathrm{~m} / \mathrm{s}$ a las $10 \mathrm{HLV}$. Esta 
particularidad se interpreta como un posible error en la transcripción de los datos de las bandas a la forma 0027 . rasgo que debería revisarse en caso de que se pudiese tener acceso a los registros originales. El rango de la curva es de $3,1 \mathrm{~m} / \mathrm{s}$ y resalta la velocidad media sostenida de $3,9 \mathrm{~m} / \mathrm{s}$ a las $13-14$ HLV, valor que resulta el mayor promediado a lo largo del año. (Figura 13).

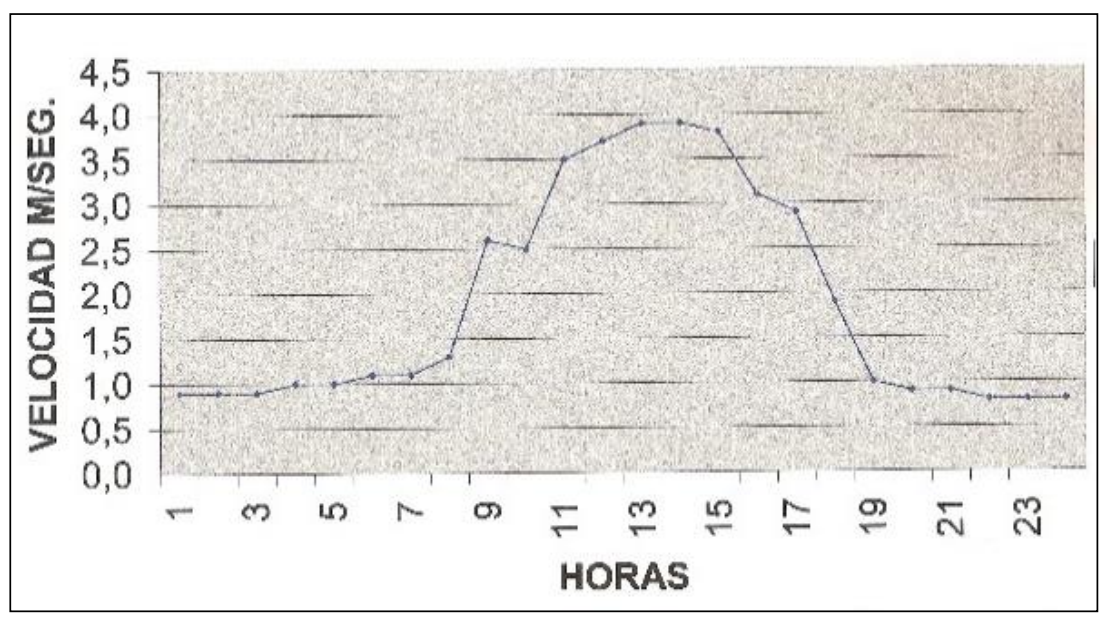

Figura 13. Variación horaria de la velocidad media del viento en el mes de octubre. Fuente: elaboración propia.

Variación de la velocidad media máxima destacada a las 14 HLV y una horaria del mes de noviembre (1982- mínima a las $20 \mathrm{HLV}$, trazos que 1991) conforma una de los regímenes

El rango de la curva del mes de horarios más regulares del año (Figura noviembre es de $3,1 \mathrm{~m} / \mathrm{s}$, con una 14).

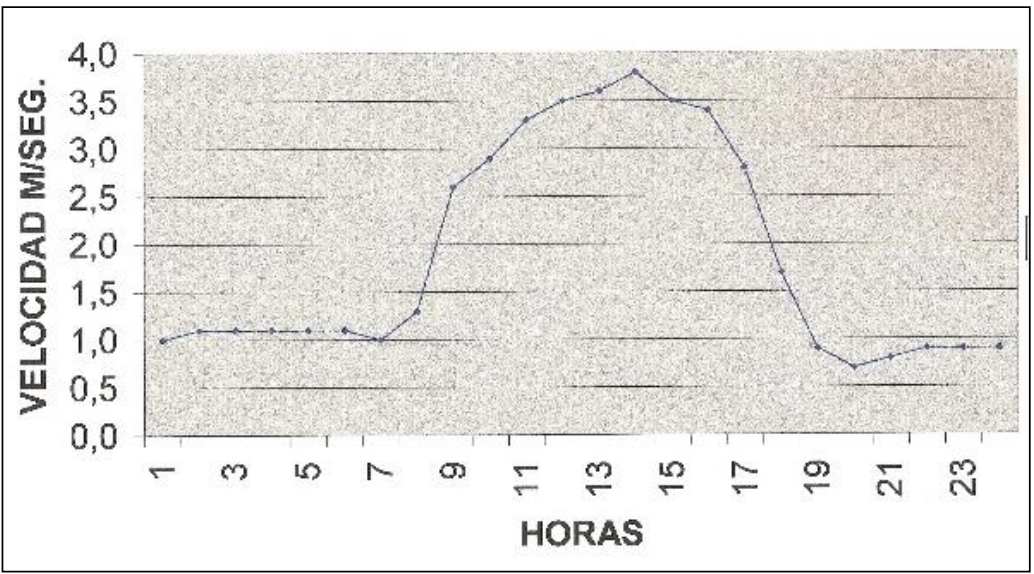

Figura 14. Variación horaria de la velocidad media del viento en el mes de noviembre. Fuente: elaboración propia. 
Variación de la velocidad media horaria del mes de diciembre (19821991)

La curva de este mes culmina a las 13-14, con medias de $3,6 \mathrm{~m} / \mathrm{s}$ y valores mínimos de $0,8-0,9 \mathrm{~m} / \mathrm{s}$, de las 19 a las
$24 \mathrm{HLV}$. Desde las 19 hasta las $08 \mathrm{HLV}$, el segmento de la curva de bajo gradiente se observa con leves cambios de tendencia a las 21, a las 04 y a las 07-08 HLV. (Figura 15).

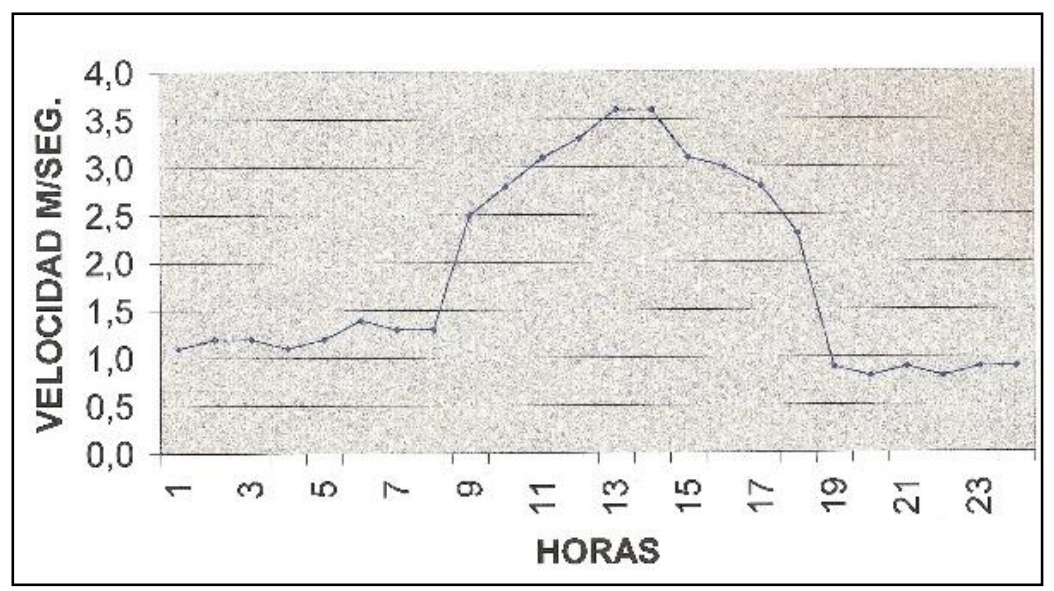

Figura 15. Variación horaria de la velocidad media del viento en el mes de diciembre. Fuente: elaboración propia.

En síntesis, se observan dos tipos diferentes de regímenes de velocidad media horaria del viento, el primero caracterizado por curvas con dos o tres picos hacia las horas centrales del día, como es el caso de los meses de enero, febrero, marzo y abril, mientras que el segundo tipo de régimen se caracteriza por curvas con una sola máxima, la cual ocurre con mayor frecuencia a las 13-14 HLV. Ambos regímenes muestran un lapso con gradientes de velocidad horaria muy bajos, en las horas finales e iniciales del día, y otro lapso con gradientes ascendentes y descendentes fuertes, pasando por la culminación de las horas centrales. Estas diferencias responden obviamente al ciclo diario de calentamiento del aire y a la consecuente activación de los gradientes báricos horizontales a escala loca.

\section{Variaciones de la dirección prevaleciente horaria del viento}

En correspondencia con las velocidades medias horarias antes descritas, las direcciones con mayor frecuencia para cada hora fueron las que se anotan seguidamente.

Variación de la dirección prevaleciente horaria de la década (1982-1991)

El examen de los registros de dirección, evidencia que desde las 23 hasta las $10 \mathrm{HLV}$ prevalece en la serie examinada la dirección NNE, compartida con la situación de calma. A las 11 y 12 HLV domina la dirección NE para dar paso a la dirección SSE prevaleciente desde las 13 hasta las 18 
$\mathrm{HLV}$. Siguen las condiciones de viento variable y calma desde las 19 hasta las $22 \mathrm{HLV}$. De esta forma, se aprecia que, en promedio, la dirección del viento recorre los cuadrantes primero y segundo, barriendo un ángulo de unos $160^{\circ}$.

Variación de la dirección prevaleciente horaria para el mes de enero

Desde la 23 hasta las 03 HLV se comparte las condiciones de calma con la dirección NNE. De las 04 a las 10 HLV domina la dirección NNE, que pasa a NE de 11 a 12 HLV para luego instaurarse la dirección SSE de las 13 a las $18 \mathrm{HLV}$, seguida de las condiciones de calma y variable hasta las $22 \mathrm{HLV}$.

Variación de la dirección prevaleciente horaria para el mes de febrero

Entre las 19 y las $07 \mathrm{HLV}$ comparten las mayores frecuencias la condición de calma y las direcciones N, NNE y NE. Entre las 09 y las $12 \mathrm{HLV}$ dominan los vientos del NNE y NE. A partir de las 13 $\mathrm{HLV}$ el viento gira al segundo cuadrante y sopla del SE hasta las 18 HLV.

Variación de la dirección prevaleciente horaria para el mes de marzo

Para el mes de marzo hay que resaltar la notoria disminución de la frecuencia de los vientos del segundo cuadrante, los cuales aparecen como dominante sólo desde la 15 a las $18 \mathrm{HLV}$, para dar paso a condiciones de calma y viento variable, compartido con la dirección NE hasta las $24 \mathrm{HLV}$. Desde la 01 a las $13 \mathrm{HLV}$ prevalece la dirección NNE y a las 14 aparecen dominantes vientos del ENE y flujos variables, lo que evidencia el giro hacia el segundo cuadrante.

Variación de la dirección prevaleciente horaria para el mes de abril

Desde las 12 hasta las $19 \mathrm{HLV}$ dominan vientos del SE y ESE. A las 20 HLV se inicia el giro hacia el primer cuadrante, con componentes del ESE y ENE, giro que continúa de las 21 a las 24 HLV, con flujos del NE, para dar paso a la dirección NNE que prevalece a partir de las 01 hasta 08 HLV, seguida de vientos de flujos variables y direcciones NE y ENE hasta las 11 HLV.

Variación de la dirección prevaleciente horaria para el mes de mayo

En mayo, el dominio del segundo cuadrante se inicia a las $11 \mathrm{HLV}$ como ESE/SSE y continúa hasta las $16 \mathrm{HLV}$, como SSE, para pasar a $S\left(180^{\circ}\right)$ a las 17 y $18 \mathrm{HLV}$ y luego a condiciones de calma y viento variable hasta las $03 \mathrm{HLV}$, cuando la condición de calma se comparte con direcciones del NNE, dominando éstas sólo de 08 a $09 \mathrm{HLV}$, seguidas por viento del NE a las $10 \mathrm{HLV}$. Durante este mes se observa 
una clara disminución de la frecuencia de las componentes del primer cuadrante.

Variación de la dirección prevaleciente horaria para el mes de junio

Similarmente al mes anterior, en junio las componentes del primer cuadrante son minoritarias, siendo prevalecientes sólo entre las 09 y las $10 \mathrm{HLV}$. Desde las 11 hasta las 19 HLV domina netamente la dirección SSE, seguida por condiciones de calma y flujos variables el resto del período.

Variación de la dirección prevaleciente horaria para el mes de julio

Las direcciones del segundo cuadrante ESE y SSE prevalecen entre las 11 y las $16 \mathrm{HLV}$, para dar paso a flujos variables y luego a condiciones de calma hasta las $08 \mathrm{HLV}$. La dirección NE domina únicamente a las 09, mientras que a las $10 \mathrm{HLV}$ los flujos variables indican el cambio hacia el segundo cuadrante.

Variación de la dirección prevaleciente horaria para el mes de agosto

Entre las 12 y las $16 \mathrm{HLV}$ domina la dirección SSE, para luego dar paso a flujos variables y condiciones de calma hasta las $11 \mathrm{HLV}$. En este mes las direcciones del primer cuadrante no llegan a ser dominantes a ninguna hora.
Variación de la dirección prevaleciente horaria para el mes de septiembre

Aparece como dominante la dirección ENE a las 09 HLV; seguidamente el viento pasa a soplar del SSE hasta las $16 \mathrm{HLV}$ y luego se presentan direcciones variables compartidas con condiciones de calma hasta las 03 y calmas con componentes $\mathrm{N}$ hasta las $08 \mathrm{HLV}$.

Variación de la dirección prevaleciente horaria para el mes de octubre

La dirección SSE domina sin interrupción desde las 11 hasta las 18 HLV. Seguidamente se instalan condiciones de viento variable y calma hasta las 24. Desde las 01 a las 09 comparten frecuencias las calmas con flujos del NNE y a las 10 la dirección ESE evidencia el cambio del viento hacia el segundo cuadrante.

Variación de la dirección prevaleciente horaria para el mes de noviembre

Como en el mes anterior, se aprecia el dominio de vientos del SSE desde las 11 hasta las $18 \mathrm{HLV}$. Sigue igualmente un período de vientos variables y calmas hasta las $04 \mathrm{HLV}$, para dar paso a flujos del NNE compartidos con calmas hasta las $10 \mathrm{HLV}$.

Variación de la dirección prevaleciente horaria para el mes de diciembre

Las direcciones del segundo cuadrante se activan a las 12 como ESE y luego SE a las 13, para pasar a SSE desde las 14 a las 20. Entre las 21 y la 01 
las condiciones de calma alternan con vientos variables. A las 03 aparecen flujos del NNE compartidos con calmas y a las $11 \mathrm{HLV}$, los vientos prevalecientes del ENE y del E, evidencian el cambio gradual hacia el segundo cuadrante.

\section{DISCUSIÓN}

Como se evidencia en las descripciones anteriores, durante el período 1982-1991, en la estación de Santa Elena de Uairén los vientos prevalecientes en el ciclo diario se reparten entre el primero y el segundo cuadrantes, con componente zonal este y diferentes números de horas de prevalencia según la época del año.

Al respecto es de interés resaltar que en ningún mes se presentan lapsos horarios en los cuales dominen flujos de los cuadrantes tercero y cuarto, de componente zonal oeste, como ocurría en el antiguo emplazamiento de la estación meteorológica, según fue demostrado en un estudio de la serie temporal 1971-1980, en el que se evidenció que durante los meses de mayo a octubre, junto con el aumento del porcentaje de calmas y disminución de la frecuencia de vientos del primer cuadrante, aparece una considerable frecuencia de flujos de los cuadrantes tercero y cuarto, de manera que ciertos días el viento realiza un giro diario de casi 360 grados (Foghin-Pillin y Reyes 1997, p. 27).

Las diferencias entre las direcciones prevalecientes registradas en las dos series temporales parecen evidenciar la influencia de los cercanos relieves montañosos que rodeaban la estación en su emplazamiento antiguo, a diferencia de las condiciones topográficas más abiertas en las que se ubica la estación desde su traslado cerca del aeropuerto, en 1981. Esta explicación también encuentra soporte en la comparación de los porcentajes de calmas entre la estación antigua y la estación actual (Tabla 1).

En la Tabla 1 se observan los porcentajes de calma notablemente mayores en el antiguo emplazamiento de la estación, rodeada de relieves conspicuos poco distantes de la localidad, en comparación con la topografía relativamente llana del emplazamiento actual, datos consistentes con las componentes vectoriales del viento en superficie, específicamente con la mayor fuerza de rozamiento que experimentan los vientos en áreas montañosas. 
Tabla 1. Porcentajes de calma en la estación de Santa Elena de Uairén durante las décadas 1971-1980 (antiguo emplazamiento) y 1982-1991 (emplazamiento actual).

\begin{tabular}{lccc}
\cline { 2 - 3 } & $\mathbf{1 9 7 1 - 1 9 8 0}$ & $\mathbf{1 9 8 2 - 1 9 9 1}$ & Diferencia \\
\hline Enero & $16,8 \%$ & $11,0 \%$ & $5,8 \%$ \\
Abril & $22,8 \%$ & $12,4 \%$ & $10,4 \%$ \\
Julio & $29,5 \%$ & $21,6 \%$ & $7,9 \%$ \\
Septiembre & $37,8 \%$ & $18,4 \%$ & $19,4 \%$ \\
\hline
\end{tabular}

Fuente: elaboración propia.

CONCLUSIONES

El análisis de la serie temporal de registros anemométricos 1982-1991 de la estación de Santa Elena de Uairén revela la alternancia diaria de componentes de los cuadrantes primero y segundo, correspondientes a ángulos acimutales entre 01 y 180 grados, con nula prevalencia de direcciones correspondientes a los cuadrantes tercero y cuarto.

Las direcciones pertenecientes al primer segundo tienden a prevalecer en las horas centrales del día, en correspondencia con las velocidades medias horarias más altas, mientras que las direcciones del primer cuadrante se presentan hacia las horas iniciales y finales del ciclo diario, en correspondencia con las velocidades más bajas del ciclo, compartidas con frecuentes períodos de calma.

La comparación de esta serie (19821991) con los registros anemométricos de la serie temporal 1971-1980 revela notables diferencias por cuanto en la antigua ubicación de la estación, durante los meses que corresponden al avance septentrional de la convergencia intertropical (CIT) se registraron variaciones diarias del viento de casi 360 grados. Sin embargo, el aumento de la frecuencia de los flujos diarios del segundo cuadrante durante el período de influencia CIT, no se interpreta como avance septentrional de los alisios de sureste, sino como producto del aumento del porcentaje de calmas, lo cual permite la activación de los gradientes báricos horizontales a escala local.

Estas variaciones determinadas por el relieve local han de tomarse en consideración en la planificación de la orientación de las pistas de aterrizaje, como se observa al comparar los casos de los aeródromos de Pacaraima, en teritorio brasileño, cuya pista se orienta 31 - 13, en comparación con la de Santa Elena, con una orientación 29 - 11 (observación propia).

Se destaca la urgencia de rescatar los registros anemométricos originales (bandas de anemógrafo y libretas diarias de observación meteorológica, entre otros) con la finalidad de recuperar registros que permitan evaluar la 
influencia que pueden tener fenómenos meteorológicos de extraordinaria magnitud, como los denominados downbursts, sobre las medias de velocidad, así como las direcciones más frecuentes de los vientos en tales condiciones, las cuales pueden representar altos riesgos sobre todo para las operaciones aeronáuticas.

De igual modo, resulta urgente solicitar ante las autoridades competentes la digitalización de todos los registros climatológicos, tanto para preservarlos como para facilitar futuras investigaciones de alto interés para diferentes áreas de la ingeniería y de las ciencias ambientales en general.

\section{REFERENCIAS BIBLIOGRÁFICAS}

Barros, V. R. y Schmidt, I. G. (1988). On the extension of climatic series from short records. Journal of Applied Meteorology, 27, 325-335. Recuperado de https://journals.ametsoc.org/doi/pd f/10.1175/1520.0450(1988)027\%3C0325:OTEOCS\%3E 2.0.CO\%3B2

Barry, R. (1992). Mountain weather and climate. London: Routledge

Benaim-Pinto, C. (1972). Pollen of Melinis minutiflora Beauv (molasses grass): An airborne biological pollutant with possible implication in the etiology of respiratory allergy; preliminary report. Acta Científica Venezolana, 23, 155-156

Burnet, M. y White, D. O. (1982). Historia natural de la enfermedad infecciosa. Madrid: Alianza Editorial

Dirección de Cartografía Nacional. (1977). Santa Elena de Uairén, Venezuela. Hoja 7929, escala
1:100.000. Caracas: Ministerio del Ambiente y de los Recursos Naturales Renovables

Escobar, D. F., Silveira, F, A. y Morellato, L. P. (2018). Timing of seed dispersal and seed dormancy in Brazilian savanna: two solutions to face seasonality. Annals of Botany, 121 (6), 1197-1209. Recuperado de https://watermark.silverchair.com/mc y006.pdf

Ezike, D. N., Nnamani, C. V., Ogundipe, O. T. y Adekanmbi, O. H. (2016). Airborne pollen and fungal spores in Garki, Abuja (North-Central Nigeria). Aerobiología, 32(4), 697-707. Recuperado de https://link.springer.com/content/pdf /10.1007/s 10453-016-9443-5.pdf

Foghin-Pillin, S. (2002). Tiempo y Clima en Venezuela. Caracas: Universidad Pedagógica Experimental Libertador Foghin-Pillin, S. y Reyes, V. M. (1997). Descripción del régimen eólico diario 1971-1980 en la estación de Santa Elena de Uairén. Ponencia $V$ Jornada Anual de Investigación (p. 27). Instituto Pedagógico de Caracas, Universidad Experimental Libertador

Freile, A. (1962). Mapa fisiográfico de la República de Venezuela. Caracas: Ministerio de Minas e Hidrocarburos

Guevara D., J. M. (1987). Métodos de estimación y ajuste de datos climáticos. Caracas: Universidad Central de Venezuela

Hafner, W. D. y Hites, R. A. (2005). Effects of Wind and Air Trajectory Directions on Atmospheric Concentrations of Persistent Organic Pollutants near the Great Lakes. Environmental Science and Technology, 39(20), 7817-7825. Recuperado de https://pubs.acs.org/doi/pdf/10.1021 /es0502223 
Henry, J. G. y Heinke, G. W. (1999). Ingeniería ambiental. México: Prentice Hall/Pearson.

Jiménez, P. A., González-Rouco, J. F., Montávez, J. P., García-Bustamante, E. y Navarro, J. (2009). Climatology of wind patterns in the northeast of the Iberian Peninsula. International Journal of Climatology, 29(4), 501525. Recuperado de https://rmets.onlinelibrary.wiley.com /doi/epdf/10.1002/joc.1705

Jiménez S., J. G. (2018). The Orinoco Low-level jet. [Tesis doctoral, The Pennsylvania State University]. Pennsylvania, EEUU

Kaczmarek, J., Kedziora, A., Brachaczek, A., Latunde-Dada, A.O., Dakowska. S., Karg, G. y Jedryczka, M. (2016). Effect of climate change on sporulation of the teleomorphs of Leptosphaeria species causing stem canker of brassicas. Aerobiologia, 32 (1), 39-51. Recuperado de https://link.springer.com/content/pd f/10.1007\%2Fs 10453-015-9404-4.pdf

Kitada, T., Okamura, K. y Tanaka, S. (1998). Effects of Topography and Urbanization on Local Winds and Thermal Environment in the Nohbi Plain, Coastal Region of Central Japan: A Numerical Analysis by Mesoscale Meteorological Model with a $k-\varepsilon$ Turbulence Model. Journal of Applied Meteorology, 37(10), 1026-1046. Recuperado de https://journals.ametsoc.org/doi/pd f/10.1175/1520-
0450\%281998\%29037\%3C 1026\%3AEO TAUO\%3E2.0.CO\%3B2

Miller, S. T. K., Keim, B. D., Talbot, R. W., y Mao, H. (2003). Sea breeze: structure, forecasting and impacts. Reviews of geophysics, 41 (3): 1-31. Recuperado de https://agupubs.onlinelibrary.wiley.c om/doi/pdf/10.1029/2003RG000124

Pino, A. y Espinosa, J. (2001). Datos meteorológicos en la cuenca del canal de Panamá durante el siglo XIX y su aplicabilidad a la variabilidad climática y al cambio climático. Tecnociencia, 3(2), 21-45. Recuperado de https://revistas.up.ac.pa/index.php/ tecnociencia/article/view/547/456.

Schubert, C. y Huber, O. (1989). La Gran Sabana: Panorámica de una región. Caracas: Lagoven

Serafin S. y Zardi, D. (2010). Daytime Heat Transfer Processes Related to Slope Flows and Turbulent Convection in an Idealized Mountain Valley. Journal of the Atmospheric Sciences, 67(11), 37393756. Recuperado de https://journals.ametsoc.org/doi/pd f/10.1175/2010JAS3428.1

Servicio de Meteorología de la Fuerza Aérea Venezolana-SMFAV. (1993). Estadísticas climatológicas de Venezuela. Período 1961-1990. Maracay: SMFAV

Simpson, J. (1994). Sea breeze and local winds. Cambridge: Cambridge University Press 